Research Article

\title{
Hermite-Hadamard, Jensen, and Fractional Integral Inequalities for Generalized $P$-Convex Stochastic Processes
}

\author{
Fangfang $M a \mathbb{D}^{1},{ }^{1}$ Waqas Nazeer $\mathbb{D}^{2},{ }^{2}$ and Mamoona Ghafoor ${ }^{3}$ \\ ${ }^{1}$ Department of Foundational Course, Shandong University of Science and Technology, Taian 271019, China \\ ${ }^{2}$ Department of Mathematics, GC University, Lahore, Pakistan \\ ${ }^{3}$ Department of Mathematics, University of Okara, Okara, Pakistan \\ Correspondence should be addressed to Fangfang Ma; sdustmff@163.com and Waqas Nazeer; nazeer.waqas@gmail.com
}

Received 22 January 2021; Accepted 23 June 2021; Published 13 July 2021

Academic Editor: Ahmet Ocak Akdemir

Copyright (C) 2021 Fangfang Ma et al. This is an open access article distributed under the Creative Commons Attribution License, which permits unrestricted use, distribution, and reproduction in any medium, provided the original work is properly cited.

The stochastic process is one of the important branches of probability theory which deals with probabilistic models that evolve over time. It starts with probability postulates and includes a captivating arrangement of conclusions from those postulates. In probability theory, a convex function applied on the expected value of a random variable is always bounded above by the expected value of the convex function of that random variable. The purpose of this note is to introduce the class of generalized $p$-convex stochastic processes. Some well-known results of generalized $p$-convex functions such as Hermite-Hadamard, Jensen, and fractional integral inequalities are extended for generalized $p$-stochastic convexity.

\section{Introduction}

A stochastic process is a mathematical tool commonly defined as a set of random variables in various fields of probability. Verifiably, random variables were related to or listed by a lot of numbers, normally as focuses in time, giving the translation of a stochastic process, speaking to numerical estimations, some systems randomly changing over time, such as the growth of bacterial populations, fluctuations in electrical flow due to thermal noise, or the production of gas molecules. Stochastic systems are commonly used as scientific models of systems that tend to alter in an arbitrary manner. They have applications in various fields, especially in sciences, for instance, chemistry, physics, biology, neuroscience, and ecology, in addition to technology and engineering fields, for example, picture preparing, cryptography, signal processing, telecommunications, PC science, and data theory. Furthermore, apparently, arbitrary changes in money-related markets have inspired the broad utilization of stochastic processes in fund.

Convex stochastic processes and their applications have a fundamental significance in mathematics and in probability. Nikodem [1] in 1980 proposed the idea of convex stochastic processes in his article. In 1992, $\lambda$-convex and Jensen-convex stochastic processes were initiated by Skowronski [2]. More recently, Kotrys presented in [3] the results on convex stochastic processes.

For more details, refer to [4]. Many studies in the literature have been performed on some extensions of convex stochastic processes and on Hermite-Hadamard type inequalities for these extensions [5].

In the present note, we purpose to investigate the idea of generalized $p$-convex stochastic processes. The notion of inequality as convexity has a significant place in literature [6], as it yields a broader setting in order to investigate the mathematical programming and optimization problems. Therefore, Schur type, Hermite-Hadamard, Jensen, and fractional integral inequalities and some important results for the above said processes will be obtained in this study.

We start by definition of the stochastic process [7].

Definition 1 (see [3]). Assume a probability space $(\Omega, \mathscr{A}, P)$. A random variable is the function $\xi: \Omega \longrightarrow \mathbb{R}$ if $\xi$ is $\mathscr{A}$-measurable; whereas, a stochastic process is the function $\xi: I \times \Omega \longrightarrow \mathbb{R}$ if $\xi(t,$.$) is a random variable for every t \in I$. 
Let us review some basic notions about stochastic processes.

Definition 2 (see [3]). The stochastic process $\xi: I \times \Omega \longrightarrow \mathbb{R}$ is as follows:

(1) Continuous on $I$, if for all $u_{\circ} \in I$,

$$
P-\lim _{u \longrightarrow u_{\circ}} \xi(u, .)=\xi\left(u_{\circ}, .\right) \text {, }
$$

where $P$ - lim represents the limit in the probability;

(2) Mean-square continuous on $I$, if for every $u_{\circ} \in I$,

$$
\lim _{u \longrightarrow u_{\circ}} \mathbb{E}\left(\xi(u, .)-\xi\left(u_{\circ}, .\right)\right)^{2}=0,
$$

Where $\mathbb{E}[\xi(u,)$.$] represents an expectation of the$ random variable $\xi$.

It is obvious in probability that if a stochastic process is mean-square continuous, then it is also continuous, but the converse is not true;

(3) Mean square is differentiable at $t \in I$, if there is a random variable $\xi^{\prime}(\nu,):. I \times \Omega \longrightarrow \mathbb{R}$, such that for every $u_{\circ} \in I$,

$$
\xi^{\prime}\left(u_{\circ}, .\right)=P-\lim _{u \longrightarrow u_{\circ}} \frac{\xi(u, .)-\xi\left(u_{\circ}, .\right)}{u-u_{\circ}} .
$$

Definition 3 (see [3]). Consider $\xi: I \times \Omega \longrightarrow \mathbb{R}$, a stochastic process with $\mathbb{E}\left[\xi(t, .)^{2}\right]<\infty$. We say a random variable $v: \Omega \longrightarrow \mathbb{R}$ to be the mean-square integral of the process $\xi$ on $\left[a_{1}, a_{2}\right]$ if for each normal sequence of partitions of $\left[a_{1}, a_{2}\right], \quad a_{1}=u_{\circ}<u_{1}<\cdots<u_{n}=a_{2}, \quad$ and for all $\Theta_{k} \in\left[u_{k-1}, u_{k}\right]$, we have

$$
\lim _{n \rightarrow \infty} \mathbb{E}\left[\left(\sum_{k=1}^{n} \xi\left(\Theta_{k}, .\right)\left(u_{k}-u_{k-1}\right)-v(.)\right)^{2}\right]=0 .
$$

Then, $v($.$) is written as$

$$
\nu(.)=\int_{a_{1}}^{a_{2}} \xi(s, .) \mathrm{d} s,(\text { a.e }) .
$$

For more on mean-square integrable stochastic processes, refer [8].

Theorem 1 (see [3]). Let us consider the Jensen-convex stochastic process $\xi: I \times \Omega \longrightarrow \mathbb{R}$ that is mean-square continuous on $I$; then, we have

$$
\xi\left(\frac{a_{1}+a_{2}}{2}, .\right) \leq \frac{1}{a_{2}-a_{1}} \int_{a_{1}}^{a_{2}} \xi(u, .) \mathrm{d} u \leq \frac{\xi\left(a_{1}, .\right)+\xi\left(a_{2}, .\right)}{2}, \text { (a.e), }
$$

for all $a_{1}, a_{2} \in I, a_{1}<a_{2}$. The above inequality is HermiteHadamard inequality for stochastic convexity.

Let us present some important generalizations of convex stochastic processes.
Definition 4 (see [9]). A stochastic process $\xi: I \times \Omega \longrightarrow \mathbb{R}$ is said to be generalized convex if for $\theta \in[0,1]$ and $u, v \in I$,

$$
\xi(\theta u+(1-\theta) v, .) \leq \xi(v, .)+\theta \eta(\xi(u, .), \xi(v, .)), \text { (a.e). }
$$

Definition 5 (see [10]). An interval $I$ is a $p$-convex set if $\left[\theta u^{p}+(1-\theta) v^{p}\right]^{(1 / p)} \in I$ for all $u, v \in I, \quad \theta \in[0,1]$, and $p=2 m+1$ or $p=(r / n), r=2 s+1, n=2 t+1$, and $m, s, t \in N$.

Definition 6 (see [10]). A function $f: I \longrightarrow \mathbb{R}$ is $p$-convex, if for $\theta \in[0,1]$ and $u, v \in I$, we have

$$
f\left(\left[\theta u^{p}+(1-\theta) v^{p}\right]^{(1 / p)}\right) \leq \theta f(u)+(1-\theta) f(v),
$$

where $I$ is a $p$-convex set.

Remark 1 (see [11]). If $I \subset(0, \infty)$ be a real interval and $p \in \mathbb{R}\{0\}$, then

$$
\left[t x^{p}+(1-t) y^{p}\right]^{(1 / p)} \in I, \quad \text { for all } x, y \in I \text { and } t \in[0,1] .
$$

According to Remark 1, we can give a different version of the definition of $p$-convex function as follows:

Definition 7 (see [11]). If $I \subset(0, \infty)$ be a real interval and $p \in \mathbb{R}\{0\}$. A function $f: I \longrightarrow \mathbb{R}$ is said to be a $p$-convex function if

$$
f\left(\left[t x^{p}+(1-t) y^{p}\right]^{(1 / p)}\right) \leq t f(x)+(1-t) f(y),
$$

for all $x, y \in I$ and $t \in[0,1]$. If the inequality (10) is reversed, then $f$ is said to be $p$-concave.

Definition 8 (see [12]). A process $\xi: I \times \Omega \longrightarrow \mathbb{R}$, where $I$ is a $p$-convex set, is said to be a $p$-convex stochastic process if for $\theta \in[0,1]$ and $u, v \in I$, we have

$$
\xi\left(\left[\theta u^{p}+(1-\theta) v^{p}\right]^{(1 / p)}, .\right) \leq \theta \xi(u, .)+(1-\theta) \xi(v, .), \text { (a.e). }
$$

In [13], the following functions are defined.

\section{Definition 9}

$$
\beta(u, v)=\int_{0}^{1} \theta^{u-1}(1-\theta)^{v-1} \mathrm{~d} \theta, \quad u, v>0 .
$$

Definition 10. For $w>v>0,|z|<1$,

$$
{ }_{2} F_{1}(u, v ; w ; z)=\frac{1}{\beta(v, w-v)} \int_{0}^{1} \theta^{v-1}(1-\theta)^{w-v-1}(1-z \theta)^{-u} \mathrm{~d} \theta .
$$

Now, we are in position to define the main notion of this article.

Definition 11. A stochastic process $\xi: I \times \Omega \longrightarrow \mathbb{R}$ is said to be generalized $p$-convex, if for $\theta \in[0,1]$ and, we have 


$$
\xi\left(\left[\theta u^{p}+(1-\theta) v^{p}\right]^{(1 / p)}, .\right) \leq \xi(v, .)+\theta \eta(\xi(u, .), \xi(v, .)), \text { (a.e) }
$$

If the inequality in (14) is reversed, then $\xi$ is the generalized $p$-concave.

Remark 2. It is obvious that the inequality (14) reduces to the convex stochastic process for $p=1$ and $\eta(x, y)=x-y$.

Example 1. Consider a stochastic process $\xi:(0, \infty) \times$ $\Omega \longrightarrow \mathbb{R}$ defined by $\xi(u,)=.u^{p}, p \neq 0$, and $\eta(x, y)=x-y$; then, $\xi$ is the generalized $p$-convex.

We organize our study as follows. First we derive some basic properties for this generalization. In next section, Schur type inequality is obtained. The third, fourth, and fifth sections are devoted to Hermite-Hadamard, Jensen, and fractional integral inequalities for generalized $p$-convex stochastic processes.

Proposition 1. Let $\xi_{1}, \xi_{2}: I \times \Omega \longrightarrow \mathbb{R}$ be two generalized p-convex stochastic processes:

(1) If $\eta$ is additive, then $\xi_{1}+\xi_{2}: I \times \Omega \longrightarrow \mathbb{R}$ is also a generalized $p$-convex stochastic process

(2) If $\eta$ is nonnegatively homogeneous, then $\lambda \xi_{1}$ : $I \times \Omega \longrightarrow \mathbb{R}$, for any $\lambda \geq 0$, is the generalized p-convex stochastic process

The proof Proposition 1 is straightforward.

Theorem 2. Assume a nonempty collection $\left\{\xi_{j}: I \times \Omega \longrightarrow \mathbb{R}, j \in J\right\}$ of generalized p-convex stochastic processes, such that

(1) There exist $\alpha \in[0, \infty]$ and $\beta \in[-1, \infty]$, such that $\eta(u, v)=\alpha u+\beta v$ for all $u, v \in \mathbb{R}$

(2) For each $u \in I, \max _{j \in J} \xi_{j}(u,$.$) exists in \mathbb{R}$; then, the stochastic process defined by $\xi(u,)=.\max _{j \in J} \xi_{j}(u,$. for all $u \in I$ is the generalized $p$-convex.

Proof. For any $u, v \in I$ and $\theta \in[0,1]$, we have

$$
\begin{aligned}
\xi\left(\left[\theta u^{p}+(1-\theta) v^{p}\right]^{(1 / p)}, .\right) & =\max _{j \in J} \xi_{j}\left(\left[\theta u^{p}+(1-\theta) v^{p}\right]^{(1 / p)}, .\right) \\
& \leq \max _{j \in J}\left\{\xi_{j}(v, .)+\theta \eta\left(\xi_{j}(u, .), \xi_{j}(v, .)\right)\right\} \\
& =\max _{j \in J}\left\{\xi_{j}(v, .)+\theta\left(\alpha \xi_{j}(u, .)+\beta \xi_{j}(v, .)\right)\right\} \\
& =\max _{j \in J}\left\{(1+\beta \theta) \xi_{j}(v, .)+\alpha \theta \xi_{j}(u, .)\right\} \\
& \leq(1+\beta \theta) \max _{j \in J} \xi_{j}(v, .)+\alpha \theta \max _{j \in J} \xi_{j}(u, .) \\
& =(1+\beta \theta) \xi(v, .)+\alpha \theta \xi(u, .) \\
& =\xi(v, .)+\theta(\alpha \xi(u, .)+\beta \xi(v, .)) \\
& =\xi(v, .)+\theta \eta(\xi(u, .), \xi(v, .)),
\end{aligned}
$$

\section{Schur Type Inequality}

Theorem 3. For $I \subset(0, \infty)$ and $p>0$, let $\xi: I \times \Omega \longrightarrow \mathbb{R}$ is the generalized p-convex stochastic process. Then, $\forall u_{1}, u_{2}, u_{3} \in I$, such that $u_{1}<u_{2}<u_{3}$ and $u_{3}^{p}-u_{1}^{p}, u_{3}^{p}-$ $u_{2}^{p}, u_{2}^{p}-u_{1}^{p} \in(0,1)$, and we have

$$
\begin{aligned}
\xi\left(u_{3}, .\right)\left(u_{3}^{p}-u_{1}^{p}\right) & -\xi\left(u_{2}, .\right)\left(u_{3}^{p}-u_{1}^{p}\right) \\
& +\left(u_{3}^{p}-u_{2}^{p}\right) \eta\left(\left(\xi\left(u_{1}, .\right), \xi\left(u_{3}, .\right)\right) \geq 0\right. \text {, (a.e). }
\end{aligned}
$$

Proof. Let $u_{1}, u_{2}, u_{3} \in I$ be given. Then, we can easily see that

$$
\begin{aligned}
& \frac{u_{3}^{p}-u_{2}^{p}}{u_{3}^{p}-u_{1}^{p}}, \frac{u_{2}^{p}-u_{1}^{p}}{u_{3}^{p}-u_{1}^{p}} \in(0,1), \\
& \frac{u_{3}^{p}-u_{2}^{p}}{u_{3}^{p}-u_{1}^{p}}+\frac{u_{2}^{p}-u_{1}^{p}}{u_{3}^{p}-u_{1}^{p}}=1 .
\end{aligned}
$$

Setting $\theta=\left(u_{3}^{p}-u_{2}^{p} /\left(u_{3}^{p}-u_{1}^{p}\right)\right), u_{2}^{p}=\theta u_{1}^{p}+(1-\theta) u_{3}^{p}$. As $\xi$ is generalized $p$-convex, so

$$
\xi\left(u_{2}, .\right) \leq \xi\left(u_{3}, .\right)+\frac{u_{3}^{p}-u_{2}^{p}}{u_{3}^{p}-u_{1}^{p}} \eta\left(\left(\xi\left(u_{1}, .\right), \xi\left(u_{3}, .\right)\right)\right.
$$

By assuming $u_{3}^{p}-u_{1}^{p}>0$ and multiplying the above inequality by $u_{3}^{p}-u_{1}^{p}$, we get inequality (16).

\section{Hermite-Hadamard Type Inequality}

Theorem 4. For $I \subset(0, \infty)$ and $p>0$, let a mean-square generalized p-convex stochastic process $\xi:\left[u_{1}, u_{2}\right]$ $\times \Omega \longrightarrow \mathbb{R}$, which is integrable. Then, for any $u_{1}, u_{2} \in I$, $\left(u_{1}<u_{2}\right)$, the following inequality holds almost everywhere:

$$
\begin{aligned}
& \xi\left(\left[\frac{u_{1}^{p}+u_{2}^{p}}{2}\right]^{(1 / p)}, .\right)-\frac{p}{2\left(u_{2}^{p}-u_{1}^{p}\right)} \int_{u_{1}}^{u_{2}} x^{p-1} \eta \\
& \left(\left(\xi\left[u_{1}^{p}+u_{2}^{p}-x^{p}\right]^{(1 / p)}, .\right), \xi(x, .)\right) \mathrm{d} x \\
& \leq \frac{p}{u_{2}^{p}-u_{1}^{p}} \int_{u_{1}}^{u_{2}} x^{p-1} \xi(x, .) \mathrm{d} x \\
& \leq \frac{\xi\left(u_{1}, .\right)+\xi\left(u_{2}, .\right)}{2}+\frac{1}{4}\left[\eta\left(\xi\left(u_{1}, .\right), \xi\left(u_{2}, .\right)\right)\right. \\
& \left.+\eta\left(\xi\left(u_{2}, .\right), \xi\left(u_{1}, .\right)\right)\right] .
\end{aligned}
$$

Proof. Take $x^{p}=\theta u_{1}^{p}+(1-\theta) u_{2}^{p} \quad$ and $\quad y^{p}=(1-\theta)$ $u_{1}^{p}+\theta u_{2}^{p}$; so,

$$
\xi\left(\left[\frac{u_{1}^{p}+u_{2}^{p}}{2}\right]^{(1 / p)}, .\right)=\xi\left(\left[\frac{x^{p}+y^{p}}{2}\right]^{(1 / p)}, .\right) .
$$

which is as required. 
Since $\xi$ is the generalized $p$-convex, so we have

$$
\begin{aligned}
\xi\left(\left[\frac{u_{1}^{p}+u_{2}^{p}}{2}\right]^{(1 / p)}, .\right)= & \xi\left(\frac{1}{2}\left(\left[\theta u_{1}^{p}+(1-\theta) u_{2}^{p}\right]^{(1 / p)}, .\right)^{p}\right. \\
& \left.+\frac{1}{2}\left(\left((1-\theta) u_{1}^{p}+\theta u_{1}^{p}\right)^{(1 / p)}\right)^{p}\right) \\
\leq & \xi\left(\left[(1-\theta) u_{1}^{p}+\theta u_{2}^{p}\right]^{(1 / p)}, .\right) \\
& +\frac{1}{2} \eta\left(\xi\left(\left[\theta u_{1}^{p}+(1-\theta) u_{2}^{p}\right]^{(1 / p)}, .\right),\right. \\
& \left.\xi\left(\left[(1-\theta) u_{1}^{p}+\theta u_{2}^{p}\right]^{(1 / p)}, .\right)\right) .
\end{aligned}
$$

Integrating w.r.t " $\theta$," the above inequality on $[0,1]$,

$$
\begin{aligned}
\xi\left(\left[\frac{u_{1}^{p}+u_{2}^{p}}{2}\right]^{(1 / p)}, .\right) \leq & \int_{0}^{1} \xi\left(\left[(1-\theta) u_{1}^{p}+\theta u_{2}^{p}\right]^{(1 / p)}, .\right) \mathrm{d} \theta \\
& +\frac{1}{2} \int_{0}^{1} \eta\left(\xi\left(\left[\theta u_{1}^{p}+(1-\theta) u_{2}^{p}\right]^{(1 / p)}, .\right),\right. \\
& \left.\xi\left(\left[\theta u_{1}^{p}+(1-\theta) u_{2}^{p}\right]^{(1 / p)}, .\right)\right) \mathrm{d} \theta
\end{aligned}
$$

which implies

$$
\begin{aligned}
& \xi\left(\left[\frac{u_{1}^{p}+u_{2}^{p}}{2}\right]^{(1 / p)}, .\right)-\frac{p}{2\left(u_{2}^{p}-u_{1}^{p}\right)} \\
& \quad \times \int_{u_{1}}^{u_{2}} x^{p-1} \eta\left(\xi\left(\left[u_{1}^{p}+u_{2}^{p}-x^{p}\right]^{(1 / p)}, .\right), \xi(x, .)\right) \mathrm{d} x \\
& \leq \frac{p}{u_{2}^{p}-u_{1}^{p}} \int_{u_{1}}^{u_{2}} x^{p-1} \xi(x, .) \mathrm{d} x .
\end{aligned}
$$

Now,

$$
\begin{aligned}
& \int_{u_{1}}^{u_{2}} x^{p-1} \xi(x, .) \mathrm{d} x \\
& =\frac{u_{2}^{p}-u_{1}^{p}}{p} \int_{0}^{1} \xi\left(\left[\theta u_{1}^{p}+(1-\theta) u_{2}^{p}\right]^{(1 / p)}, .\right) \mathrm{d} \theta \\
& \leq \frac{u_{2}^{p}-u_{1}^{p}}{p}\left(\xi\left(u_{2}, .\right)+\int_{0}^{1} \theta \eta\left(\xi\left(u_{1}, .\right), \xi\left(u_{2}, .\right)\right) \mathrm{d} \theta\right),
\end{aligned}
$$

which implies

$$
\begin{aligned}
& \frac{p}{u_{2}^{p}-u_{1}^{p}} \int_{u_{1}}^{u_{2}} x^{p-1} \xi(x, .) \mathrm{d} x \\
& \leq \xi\left(u_{2}, .\right)+\int_{0}^{1} \theta \eta\left(\xi\left(u_{1}, .\right), \xi\left(u_{2}, .\right)\right) \mathrm{d} \theta .
\end{aligned}
$$

Similarly,

$$
\begin{aligned}
& \frac{p}{u_{2}^{p}-u_{1}^{p}} \int_{u_{1}}^{u_{2}} x^{p-1} \xi(x, .) \mathrm{d} x \\
& \leq \xi\left(u_{1}, .\right)+\int_{0}^{1} \theta \eta\left(\xi\left(u_{2}, .\right), \xi\left(u_{1}, .\right)\right) \mathrm{d} \theta .
\end{aligned}
$$

Adding (25) and (26),

$$
\begin{aligned}
& \frac{p}{u_{2}^{p}-u_{1}^{p}} \int_{u_{1}}^{u_{2}} x^{p-1} \xi(x, .) \mathrm{d} x \\
& \leq \frac{\xi\left(u_{1}, .\right)+\xi\left(u_{2}, .\right)}{2} \\
& \quad+\frac{1}{4}\left[\eta\left(\xi\left(u_{1}, .\right), \xi\left(u_{2}, .\right)\right)+\eta\left(\xi\left(u_{1}, .\right), \xi\left(u_{2}, .\right)\right)\right] .
\end{aligned}
$$
(22).

Combining (23) and (27), we obtain the inequality

Remark 3. For $p=1$ and $\eta(u, v)=u-v$ in (22), we get Hermite-Hadamard inequality (6) for the convex stochastic process.

\section{Jensen Type Inequality}

The following result will be helpful in the derivation of Jensen's type inequality for the generalized $p$-convex stochastic process.

Lemma 1. Let $w_{1}, \ldots, w_{n}$ be the positive real numbers $(n \geq 2)$. Assume $\xi: I \times \Omega \longrightarrow \mathbb{R}$ be a generalized $p$-convex stochastic process and $u_{1}, u_{2}, \ldots, u_{n} \in I$; then, we have almost everywhere

$$
\begin{aligned}
& \xi\left(\left[\frac{1}{W_{n}} \sum_{i=1}^{n} w_{i} u_{i}^{p}\right]^{(1 / p)}, .\right) \\
& =\xi\left(\left[\frac{W_{n-1}}{W_{n}} \sum_{i=1}^{n-1} \frac{w_{i}}{W_{n-1}} u_{i}^{p}+\frac{w_{n}}{W_{n}} u_{n}^{p}\right]^{(1 / p)}, .\right) \\
& \leq \xi\left(u_{n}, .\right)+\frac{W_{n-1}}{W_{n}} \eta\left(\xi\left(\left[\sum_{i=1}^{n-1} \frac{w_{i}}{W_{n-1}} u_{i}^{p}\right]^{(1 / p)}, .\right), \xi\left(u_{n}, .\right)\right),
\end{aligned}
$$

where $W_{n}=\sum_{i=1}^{n} w_{i}$.

Theorem 5 (Jensen type inequality). Let $\xi: I \times \Omega \longrightarrow \mathbb{R}$ be a generalized p-convex stochastic process and $\eta: A \times B \longrightarrow \mathbb{R}$ be nondecreasing, nonnegatively sublinear in the first variable; then, we have almost everywhere

$$
\xi\left(\left[\frac{1}{W_{n}} \sum_{i=1}^{n} w_{i} u_{i}^{p}\right]^{(1 / p)}, .\right) \leq \xi\left(u_{n}, .\right)+\sum_{i=1}^{n}\left(\frac{W_{i}}{W_{n}}\right) \eta_{\xi}\left(u_{i}, u_{i+1}, \ldots, u_{n}, .\right),
$$


where $W_{n}=\sum_{i=1}^{n} w_{i}$ and $\eta_{\xi}\left(u_{i}, u_{i+1}, \ldots, u_{n},.\right)=\eta\left(\eta_{\xi}\left(u_{i}\right.\right.$, $\left.\left.u_{i+1}, \ldots, u_{n-1},.\right), \xi\left(u_{n},.\right)\right)$ and $\eta_{\xi}(u,)=.\xi(u,.) \forall u \in I$.
Proof. Since $\eta$ is nondecreasing, nonnegatively sublinear in the first variable, so using Lemma 1, we get

$$
\begin{aligned}
& \xi\left(\left[\frac{1}{W_{n}} \sum_{i=1}^{n} w_{i} x_{i}^{p}\right]^{(1 / p)}, .\right)=\xi\left(\left[\frac{w_{n}}{W_{n}} u_{n}^{p}+\sum_{i=1}^{n-1} \frac{w_{i}}{W_{n}} u_{i}^{p}\right]^{(1 / p)}, .\right) \\
& =\xi\left(\left[\frac{W_{n-1}}{W_{n}} \sum_{i=1}^{n-1} \frac{w_{i}}{W_{n-1}} \boldsymbol{u}_{i}^{p}+\frac{w_{n}}{W_{n}} \boldsymbol{u}_{n}^{p}\right]^{(1 / p)}, .\right) \\
& \leq \xi\left(u_{n}, .\right)+\frac{W_{n-1}}{W_{n}} \eta\left(\xi\left(\left[\sum_{i=1}^{n-1} \frac{w_{i}}{W_{n-1}} u_{i}^{p}\right]^{(1 / p)}, .\right), \xi\left(u_{n}, .\right)\right) \\
& =\xi\left(u_{n}, .\right)+\frac{W_{n-1}}{W_{n}} \eta\left(\xi\left(\left[\frac{W_{n-2}}{W_{n-1}} \sum_{i=1}^{n-2} \frac{w_{i}}{W_{n-2}} u_{i}^{p}+\frac{w_{n-1}}{W_{n-1}} u_{n-1}^{p}\right]^{(1 / p)}, .\right), \xi\left(u_{n}, .\right)\right) \\
& \leq \xi\left(u_{n}, .\right)+\frac{W_{n-1}}{W_{n}} \eta\left(\xi\left(u_{n-1}, .\right)+\frac{W_{n-2}}{W_{n-1}} \eta \times\left(\xi\left(\left[\sum_{i=1}^{n-2} \frac{w_{i}}{W_{n-2}} u_{i}^{p}\right]^{(1 / p)}, .\right), \xi\left(u_{n-1}, .\right)\right), \xi\left(u_{n}, .\right)\right) \\
& \leq \xi\left(u_{n}, .\right)+\frac{W_{n-1}}{W_{n}} \eta\left(\xi\left(u_{n-1}, .\right), \xi\left(u_{n}\right)\right)+\frac{W_{n-2}}{W_{n}} \eta \\
& \times\left(\eta\left(\xi\left(\left[\sum_{i=1}^{n-2} \frac{w_{i}}{W_{n-2}} u_{i}^{p}\right]^{(1 / p)}, .\right), \xi\left(u_{n-1}, .\right)\right), \xi\left(u_{n}, .\right)\right) \\
& \leq \cdots \leq \xi\left(u_{n}, .\right)+\frac{W_{n-1}}{W_{n}} \eta\left(\xi\left(u_{n-1}, .\right), \xi\left(u_{n}, .\right)\right)+\frac{W_{n-2}}{W_{n}} \\
& \times \eta\left(\eta\left(\xi\left(u_{n-2}, .\right), \xi\left(u_{n}, .\right)\right), \xi\left(u_{n}, .\right)\right) \\
& \left.\left.+\cdots+\frac{W_{1}}{W_{n}} \eta\left(\eta\left(\ldots \eta\left(\xi\left(u_{1}, .\right), \xi\left(u_{2}, .\right)\right), \xi\left(u_{3}, .\right)\right) \ldots\right)\right), \xi\left(u_{n-1}, .\right), \xi\left(u_{n}, .\right)\right) \\
& =\xi\left(u_{n}, .\right)+\frac{W_{n-1}}{W_{n}} \eta_{\xi}\left(u_{n-1}, u_{n}, .\right)+\frac{W_{n-2}}{W_{n}} \eta_{\xi}\left(u_{n-2}, u_{n-1}, u_{n}, .\right) \\
& +\cdots+\frac{W_{1}}{W_{n}} \eta_{\xi}\left(u_{1}, u_{2}, \ldots, u_{n-1}, u_{n}, .\right) \\
& =\xi\left(u_{n}, .\right)+\sum_{i=1}^{n-1}\left(\frac{W_{i}}{W_{n}}\right) \eta_{\xi}\left(u_{i}, u_{i+1}, \ldots, u_{n}, .\right) .
\end{aligned}
$$




\section{Fractional Integral Inequalities}

Lemma 2 (see [12]). Assume a stochastic process $\xi: I \subset$ $(0, \infty) \times \Omega \longrightarrow \mathbb{R}$ which is mean-square differentiable on $I^{o}$ and $u_{1}, u_{2} \in I^{o}$ with $u_{1}<u_{2}$. If $\xi^{\prime} \in L\left[u_{1}, u_{2}\right]$, then we have almost everywhere

$$
\begin{gathered}
\frac{\xi\left(u_{1}, .\right)+\xi\left(u_{2}, .\right)}{2}-\frac{p}{u_{2}^{p}-u_{1}^{p}} \int_{u_{1}}^{u_{2}} \frac{\xi(x, .)}{x^{1-p}} \mathrm{~d} x \\
=\frac{u_{2}^{p}-u_{1}^{p}}{2 p} \int_{0}^{1} \frac{1-2 \theta}{\left[\theta u_{1}^{p}+(1-\theta) d^{p}\right]^{1-(1 / p)}} \\
\times \xi^{\prime}\left(\left[\theta u_{1}^{p}+(1-\theta) u_{2}^{p}\right]^{(1 / p)}, .\right) \mathrm{d} \theta .
\end{gathered}
$$

Theorem 6. For $I \subset(0, \infty)$ and $p>0$ and under the assumptions of Lemma 2 with $\left|\xi^{\prime}\right|^{q}$, a generalized $p$-convex stochastic process on $\left[u_{1}, u_{2}\right]$ for $q \geq 1$, then we have almost everywhere

$$
\begin{aligned}
& \left|\frac{\xi\left(u_{1}, .\right)+\xi\left(u_{2}, .\right)}{2}-\frac{p}{u_{2}^{p}-u_{1}^{p}} \int_{u_{1}}^{u_{2}} \frac{\xi(x, .)}{x^{1-p}} \mathrm{~d} x\right| \\
& \leq \frac{u_{2}^{p}-c^{p}}{2 p} L_{1}^{1-(1 / q)}\left[L_{1}\left|\xi^{\prime}\left(u_{2}, .\right)\right|^{q}\right. \\
& \left.\quad+L_{2}\left(\eta\left(\left|\xi^{\prime}\left(u_{1}, .\right)\right|^{q},\left|\xi^{\prime}\left(u_{2}, .\right)\right|^{q}\right)\right)\right]^{(1 / q)}
\end{aligned}
$$

where

$$
\begin{aligned}
L_{1}\left(u_{1}, u_{2} ; p\right)= & \frac{1}{4}\left(\frac{u_{1}^{p}-u_{2}^{p}}{2}\right)^{(1 / p)-1}\left[{ }_{2} F_{1}\left(1-\frac{1}{p}, 2,3: \frac{u_{1}^{p}-u_{2}^{p}}{u_{1}^{p}+u_{2}^{p}}\right)\right. \\
& \left.+{ }_{2} F_{1}\left(1-\frac{1}{p}, 2,3: \frac{u_{2}^{p}-u_{1}^{p}}{c^{p}+u_{2}^{p}}\right)\right], \\
L_{2}\left(u_{1}, u_{2} ; p\right)= & \frac{1}{24}\left(\frac{u_{1}^{p}+u_{2}^{p}}{2}\right)^{(1 / p)-1}\left[{ }_{2} F_{1}\left(1-\frac{1}{p}, 2,4: \frac{u_{1}^{p}-u_{2}^{p}}{u_{1}^{p}+u_{2}^{p}}\right)\right. \\
& \left.+{ }_{2} F_{1}\left(1-\frac{1}{p}, 2,4: \frac{u_{2}^{p}-u_{1}^{p}}{u_{1}^{p}+v^{p}}\right)\right] .
\end{aligned}
$$

Proof. By making use of Lemma 2 and power mean-integral inequality, we have

$$
\begin{aligned}
& \left|\frac{\xi\left(u_{1}, .\right)+\xi\left(u_{2}, .\right)}{2}-\frac{1}{u_{2}^{p}-u_{1}^{p}} \int_{u_{1}}^{u_{2}} \frac{\xi(x, .)}{x^{1-p}} \mathrm{~d} x\right| \\
& \leq \frac{u_{2}^{p}-u_{1}^{p}}{2 p} \int_{0}^{1}\left|\frac{1-2 \theta}{\left[\theta u_{1}^{p}+(1-\theta) u_{2}^{p}\right]^{1-(1 / p)}}\right| \\
& \quad \times\left|\xi^{\prime}\left(\left[\theta u_{1}^{p}+(1-\theta) u_{2}^{p}\right]^{(1 / p)}, .\right)\right| \mathrm{d} \theta \\
& \leq \frac{u_{2}^{p}-u_{1}^{p}}{2 p}\left(\int_{0}^{1} \frac{|1-2 \theta|}{\left[\theta u_{1}^{p}+(1-\theta) u_{2}^{p}\right]^{1-(1 / p)}} \mathrm{d} t\right)^{1-(1 / q)} \\
& \quad \times\left(\int_{0}^{1} \frac{|1-2 \theta|}{\left[\theta u_{1}^{p}+(1-\theta) u_{2}^{p}\right]^{1-(1 / p)}}\left|\xi^{\prime}\left(\left[\theta u_{1}^{p}+(1-\theta) u_{2}^{p}\right]^{(1 / p)}, .\right)\right|^{q} \mathrm{~d} \theta\right)^{(1 / q)} .
\end{aligned}
$$

Hence, by generalized $p$-convexity of $\left|\xi^{\prime}\right|^{q}$ on $\left[u_{1}, u_{2}\right]$, we have

$$
\begin{aligned}
& \left|\frac{\xi\left(u_{1}, .\right)+\xi\left(u_{2}, .\right)}{2}-\frac{1}{u_{2}^{p}-u_{1}^{p}} \int_{a}^{b} \frac{\xi(x, .)}{x^{1-p}} \mathrm{~d} x\right| \\
& \leq \frac{u_{2}^{p}-u_{1}^{p}}{2 p}\left(\int_{0}^{1} \frac{|1-2 \theta|}{\left[\theta u_{1}^{p}+(1-\theta) u_{2}^{p}\right]^{1-(1 / p)}} \mathrm{d} t\right)^{1-(1 / q)} \\
& \left(\int_{0}^{1} \frac{|1-2 \theta|\left[\left|\xi^{\prime}\left(u_{2}, .\right)\right|^{q}+\theta \eta\left(\left|\xi^{\prime}\left(u_{1}, .\right)\right|^{q},\left|\xi^{\prime}\left(u_{2}, .\right)\right|^{q}\right)\right]}{\left[\theta u_{1}^{p}+(1-\theta) u_{2}^{p}\right]^{1-(1 / p)}} \mathrm{d} t\right)^{(1 / q)} \\
& \leq \frac{u_{2}^{p}-u_{1}^{p}}{2 p} L_{1}^{1-(1 / q)}\left[L_{1}\left|\xi^{\prime}\left(u_{2}, .\right)\right|^{q}\right. \\
& \left.\quad+L_{2}\left(\eta\left(\left|\xi^{\prime}\left(u_{1}, .\right)\right|^{q},\left|\xi^{\prime}\left(u_{2}, .\right)\right|^{q}\right)\right)\right]^{(1 / q)} .
\end{aligned}
$$

It is easy to check that

$$
\begin{aligned}
& \int_{0}^{1} \frac{|1-2 \theta|}{\left[\theta u_{1}^{p}+(1-\theta) u_{2}^{p}\right]^{1-(1 / p)}} \mathrm{d} \theta=L_{1}\left(u_{1}, u_{2} ; p\right), \\
& \int_{0}^{1} \frac{|1-2 \theta| \theta}{\left[\theta u_{1}^{p}+(1-\theta) u_{2}^{p}\right]^{1-(1 / p)}} \mathrm{d} \theta=L_{2}\left(u_{1}, u_{2} ; p\right) .
\end{aligned}
$$

Remark 4. By setting $\eta\left(u_{1}, u_{2}\right)=u_{1}-u_{2}$ in (32), we get Theorem 4 of [12].

We will get the following Corollary by taking $q=1$ in (32). 
Corollary 1. If $\left|\xi^{\prime}\right|$ is generalized $p$-convex on $\left[u_{1}, u_{2}\right]$, then we have almost everywhere

$$
\begin{aligned}
& \left|\frac{\xi\left(u_{1}, .\right)+\xi\left(u_{2}, .\right)}{2}-\frac{p}{u_{2}^{p}-u_{1}^{p}} \int_{u_{1}}^{u_{2}} \xi(x, .) x^{1-p} \mathrm{~d} x\right| \\
& \left.\leq \frac{u_{2}^{p}-u_{1}^{p}}{2 p}\left[L_{1}\left|\xi^{\prime}\left(u_{2}, .\right)\right|+L_{2}\left(\eta\left(\left|\xi^{\prime}\left(u_{1}, .\right)\right|,\left|\xi^{\prime}\left(u_{2}, .\right)\right|\right)\right)\right)\right],
\end{aligned}
$$

where $L_{1}$ and $L_{2}$ are defined in Theorem 6.

Remark 5. If we take $\eta\left(u_{1}, u_{2}\right)=u_{1}-u_{2}$ in (37), then we have Corollary 4 of [12].

Theorem 7. For $I \subset(0, \infty)$ and $p>0$ and under the assumptions of Lemma 2 with $\left|\xi^{\prime}\right|^{q}$, a generalized $p$-convex stochastic process on $\left[u_{1}, u_{2}\right]$ for $1<q,(1 / r)+(1 / q)=1$, then we have almost everywhere

$$
\begin{aligned}
& \left|\frac{\xi\left(u_{1}, .\right)+\xi\left(u_{2}, .\right)}{2}-\frac{p}{u_{2}^{p}-u_{1}^{p}} \int_{u_{1}}^{u_{2}} \frac{\xi(x, .)}{x^{1-p}} \mathrm{~d} x\right| \\
& \leq \frac{u_{2}^{p}-u_{1}^{p}}{2 p}\left(\frac{1}{r+1}\right){ }^{(1 / r)} \\
& \quad\left[L_{4}\left|\xi^{\prime}\left(u_{2}, .\right)\right|^{q}+L_{5}\left(\eta\left(\left|\xi^{\prime}\left(u_{1}, .\right)\right|^{q},\left|\xi^{\prime}\left(u_{2}, .\right)\right|^{q}\right)\right)\right]^{(1 / q)},
\end{aligned}
$$

where

$$
\begin{aligned}
L_{3}= & L_{3}\left(u_{1}, u_{2} ; p ; q\right) \\
& = \begin{cases}\frac{1}{u_{1}^{q p-q}} \cdot{ }_{2} F_{1}\left(q-\frac{q}{p}, 1 ; 2 ; 1-\left(\frac{u_{2}}{u_{1}}\right)^{p}\right), & \text { if } p<0, \\
\frac{1}{u_{2}^{q p-q}} \cdot{ }_{2} F_{1}\left(q-\frac{q}{p}, 1 ; 2 ; 1-\left(\frac{u_{1}}{d}\right)^{p}\right), & \text { if } p>0,\end{cases} \\
L_{4}= & L_{4}\left(u_{1}, u_{2} ; p ; q\right) \\
& \begin{cases}\frac{1}{2 u_{1}^{q p-q}} \cdot{ }_{2} F_{1}\left(q-\frac{q}{p}, 1 ; 3 ; 1-\left(\frac{u_{2}}{u_{1}}\right)^{p}\right), & \text { if } p<0, \\
\frac{1}{2 u_{2}^{q p-q}} \cdot{ }_{2} F_{1}\left(q-\frac{q}{p}, 2 ; 3 ; 1-\left(\frac{u_{1}}{u_{2}}\right)^{p}\right), & \text { if } p>0 .\end{cases}
\end{aligned}
$$

Proof. By making use of Lemma 2, Holder's inequality, and generalized $p$-convexity of $\left|\xi^{\prime}\right|^{q}$ on $\left[u_{1}, u_{2}\right]$, we have

$$
\begin{aligned}
& \frac{\xi\left(u_{1}, .\right)+\xi\left(u_{2}, .\right)}{2}-\frac{p}{u_{2}^{p}-u_{1}^{p}} \int_{u_{1}}^{u_{2}} \frac{\xi(x, .)}{x^{1-p}} \mathrm{~d} x \\
& \leq \frac{u_{2}^{p}-u_{1}^{p}}{2 p}\left(\int_{0}^{1}|1-2 \theta|^{r} \mathrm{~d} t\right)^{(1 / r)} \\
& \quad \times\left(\int_{0}^{1} \frac{1}{\left[\theta u_{1}^{p}+(1-\theta) u_{2}^{p}\right]^{q-(q / p)}}\left|\xi^{\prime}\left(\left[\theta u_{1}^{p}+(1-\theta) u_{2}^{p}\right]^{(1 / p)}, .\right)\right|^{q} \mathrm{~d} t\right)^{(1 / q)} \\
& \leq \frac{d^{p}-u_{1}^{p}}{2 p}\left(\frac{1}{r+1}\right)^{(1 / r)} \\
& \quad \times\left(\int_{0}^{1} \frac{\left|\xi^{\prime}\left(u_{2}, .\right)\right|^{q}+\theta \eta\left(\left|\xi^{\prime}\left(u_{1}, .\right)\right|^{q},\left|\xi^{\prime}\left(u_{2}, .\right)\right|^{q}\right)}{\left[\theta u_{1}^{p}+(1-\theta) u_{2}^{p}\right]^{q-(q / p)}} \mathrm{d} \theta\right)^{(1 / q)},
\end{aligned}
$$

where an easy calculation gives

$$
\begin{aligned}
& \int_{0}^{1} \frac{1}{\left[\theta u_{1}^{p}+(1-\theta) u_{2}^{p}\right]^{q-(q / p)}} \mathrm{d} \theta=L_{3}\left(u_{1}, u_{2} ; p ; q\right), \\
& \int_{0}^{1} \frac{\theta}{\left[\theta u_{1}^{p}+(1-\theta) u_{2}^{p}\right]^{q-(q / p)}} \mathrm{d} \theta=L_{4}\left(u_{1}, d ; p ; q\right) .
\end{aligned}
$$

Substituting equations (41) and (42) into (38), the proof is completed.

Remark 6. By taking $\eta\left(u_{1}, u_{2}\right)=u_{1}-u_{2}$ in Theorem 7 , then we obtain Theorem 6 of [12].

Theorem 8. For $I \subset(0, \infty)$ and $p>0$ and under the assumptions of Lemma 2 with $\left|\xi^{\prime}\right|^{q}$, a generalized $p$-convex on $\left[u_{1}, u_{2}\right]$ for $1<q,(1 / r)+(1 / q)=1$, then we have almost everywhere

$$
\begin{aligned}
& \left|\frac{\xi\left(u_{1}, .\right)+\xi\left(u_{2}, .\right)}{2}-\frac{p}{u_{2}^{p}-u_{1}^{p}} \int_{u_{1}}^{u_{2}} \frac{\xi(x, .)}{x^{1-p}} \mathrm{~d} x\right| \\
& \leq \frac{u_{2}^{p}-u_{1}^{p}}{2 p} L_{5}^{(1 / r)}\left(\frac{1}{q+1}\right)^{(1 / q)} \\
& \quad\left(\left|\xi^{\prime}\left(u_{2}, .\right)\right|^{q}+\frac{1}{2} \eta\left(\left|\xi^{\prime}\left(u_{1}, .\right)\right|^{q},\left|\xi^{\prime}\left(u_{2}, .\right)\right|^{q}\right)\right)^{(1 / q)},
\end{aligned}
$$




$$
\begin{aligned}
L_{5} & =L_{5}\left(u_{1}, u_{2} ; p ; r\right) \\
& = \begin{cases}\frac{1}{u_{1}^{p r-r}} \cdot{ }_{2} F_{1}\left(r-\frac{r}{p}, 1 ; 2 ; 1-\left(\frac{u_{2}}{u_{1}}\right)^{p}\right), & \text { if } p<0, \\
\frac{1}{u_{2}^{p r-r}} \cdot{ }_{2} F_{1}\left(r-\frac{r}{p}, 1 ; 2 ; 1-\left(\frac{u_{1}}{u_{2}}\right)^{p}\right), & \text { if } p>0 .\end{cases}
\end{aligned}
$$

Proof. From Lemma 2, Holder's inequality, and generalized $p$-convexity of $\left|\xi^{\prime}\right|^{q}$ on $\left[u_{1}, u_{2}\right]$, we have

$$
\begin{aligned}
& \left|\frac{\xi\left(u_{1}, .\right)+\xi\left(u_{2}, .\right)}{2}-\frac{p}{u_{2}^{p}-u_{1}^{p}} \int_{u_{1}}^{u_{2}} \frac{\xi(x, .)}{x^{1-p}} \mathrm{~d} x\right| \\
& \leq \frac{u_{2}^{p}-u_{1}^{p}}{2 p}\left(\int_{0}^{1} \frac{1}{\left[\theta u_{1}^{p}+(1-\theta) u_{2}^{p}\right]^{q-(q / p)}} \mathrm{d} \theta\right)^{(1 / r)} \\
& \times\left(\int_{0}^{1}|1-2 \theta|^{q}\left|\xi^{\prime}\left(\left[\theta u_{1}^{p}+(1-\theta) u_{2}^{p}\right]^{(1 / p)}, .\right)\right|^{q} \mathrm{~d} \theta\right)^{(1 / q)} \\
& \leq \frac{u_{2}^{p}-u_{1}^{p}}{2 p} L_{6}^{(1 / r)}\left(\frac{1}{q+1}\right)^{(1 / q)} \\
& \quad\left(\left|\xi^{\prime}\left(u_{2}, .\right)\right|^{q}+\frac{1}{2}\left(\eta\left(\left|\xi^{\prime}\left(u_{1}, .\right)\right|^{q},\left|\xi^{\prime}\left(u_{2}, .\right)\right|^{q}\right)\right)\right)^{(1 / q)}
\end{aligned}
$$

where an easy calculation gives

$$
\begin{aligned}
& \int_{0}^{1} \frac{1}{\left[\theta u_{1}^{p}+(1-\theta) u_{2}^{p}\right]^{q-(r / p)}} \mathrm{d} \theta=L_{5}\left(c, u_{2} ; p ; r\right), \\
& \int_{0}^{1}|1-2 \theta|^{q} \mathrm{~d} \theta=\frac{1}{(q+1)} .
\end{aligned}
$$

Substituting (46) and (47) into (43), we obtain the required result.

\section{Conclusion}

There are many applications of stochastic processes, for instance, the Kolmogorov-Smirnoff test on equality of distributions. The other application includes sequential analysis and quickest detection. In this study, we have presented a new class of convex stochastic processes which are generalized $p$-convex and established Jensen, HermiteHadamard, and fractional integral inequalities for this class. Our conclusions are applicable, since the expected value of a random variable is consistently bounded above by the expected value of the convex function of that random variable. It will be interesting to find parallel results by using the proposed definition in this study in the setting of other fractional integrals $[14,15]$.

\section{Data Availability}

The data used to support the findings of this study are included within this article.

\section{Conflicts of Interest}

The authors declare that they have no conflicts of interest.

\section{Authors' Contributions}

Fangfang Ma validated the results, prepared the final draft of the manuscript, and arranged the funding for this study. Waqas Nazeer proved the main results. Mamoona Ghafoor wrote the first draft of the manuscript.

\section{Acknowledgments}

The study was supported by Science and Technology Nova Plan of Beijing City (Z201100006820122) and Fundamental Research Funds for the Central Universities (2020RC14).

\section{References}

[1] K. Nikodem, "On convex stochastic processes," Aequationes Mathematicae, vol. 20, p. 184197, 1980.

[2] A. Skowronski, "On some properties of $J$-convex stochastic processes," Aequationes Mathematicae, vol. 44, p. 249258, 1992.

[3] D. Kotrys, "Hermite-Hadamard inequality for convex stochastic processes," Aequationes Mathematicae, vol. 83, no. 1-2, pp. 143-151, 2012.

[4] S. S. Dragomir and C. E. M. Pearce, Selected Topics on Hermite-Hadamard Inequalities and Applications, RGMIA Monographs, Victoria University, Footscray, Australia, 2000.

[5] A. Skowronski, "On Wright-convex stochastic processes," Annales Mathematicae, vol. 9, pp. 29-32, 1995.

[6] Z. B. Fang and R. Shi, "On the $(p, h)$-convex function and some integral inequalities," Journal of Inequalities and Applications, vol. 45, 2014

[7] H. Zhou, M. S. Saleem, M. Ghafoor, and J. Li, "Generalization of-convex stochastic processes and some classical inequalities," Mathematical Problems in Engineering, vol. 2020, Article ID 1583807, 9 pages, 2020.

[8] K. Sobczyk, Stochastic Differential Equations with Applications to Physics and Engineering, Kluwer, Dordrecht, 1991.

[9] C. Yong Jung, M. S. Saleem, M. Shoaib Saleem, S. Bilal, W. Nazeer, and M. Ghafoor, "Some properties of $\eta$-convex stochastic processes," AIMS Mathematics, vol. 6, no. 1, pp. 726-736, 2021.

[10] K. S. Zhang and J. P. Wan, "p-convex functions and their properties," Pure and Applied Mathematics, vol. 23, no. 1, pp. 130-133, 2007.

[11] İ. İcan, "Ostrowski type inequalities for $p$-convex functions," New Trends in Mathematical Sciences, vol. 4, no. 3, p. 140, 2016.

[12] N. Okur, I. Işcan, and E. Yuksek Dizdar, "Hermite-Hadamard type inequalities for $p$-convex stochastic processes," $A n I n$ ternational Journal of Optimization and Control: Theories \& Applications (IJOCTA), vol. 9, no. 2, pp. 148-153, 2019.

[13] İ. İcan, "Hermite-Hadamard inequalities for $p$-convex functions," International Journal of Analysis and Applications, vol. 11, no. 2, pp. 137-145, 2016. 
[14] S. M. Kang, G. Farid, M. Waseem, S. Ullah, W. Nazeer, and S. Mehmood, "Generalized $k$-fractional integral inequalities associated with $(A, m)(\alpha, m)$-convex functions," Journal of Inequalities and Applications, vol. 2019, no. 1, pp. 1-14, 2019.

[15] G. Hong, G. Farid, W. Nazeer et al., "Boundedness of fractional integral operators containing mittag-leffler function via exponentially-convex functions," Journal of Mathematics, vol. 2020, Article ID 3584105, 7 pages, 2020. 